\title{
sciendo
}

Research Article

(C) 2018 Amelia Ngozi Odo and Samuel C. Onoh This is an open access article licensed under the Creative Commons Attribution-NonCommercial-NoDerivs License (http://creativecommons.org/licenses/by-nc-nd/3.0/).

\section{Food Hygiene Knowledge and Practices among Food Handlers in University of Nigeria, Nsukka Campus}

\author{
Amelia Ngozi Odo, PhD. \\ Samuel C. Onoh, B.Sc. \\ Department of Human Kinetics and Health Education, \\ University of Nigeria, 41001 Nsukka, \\ Enugu State, Nigeria
}

Doi: 10.2478/mjss-2018-0169

\begin{abstract}
The study determined food hygiene knowledge and practices among food handlers in University of Nigeria, Nsukka Campus. Two specific objectives, two corresponding research questions, and two null hypotheses guided the study. The study adopted a descriptive cross-sectional survey research design. The population for the study was 165 food handlers. There was no sampling done. A researchers'designed food hygiene knowledge and practices questionnaire was the instrument for data collection. Frequencies and percentages were used to answer the research questions while Chi-square (X2) statistic was used to test the hypotheses at .05 level of significance. Findings showed among others that all the respondents (100\%) who have no formal education were knowledgeable about food hygiene; 90.8 per cent of those who have primary education, 92.3 per cent of those who have secondary education and 92.3 of those who have no tertiary education had good knowledge of food hygiene. There was no significant difference in the level of food hygiene knowledge possessed by food handlers based on level of education $(P=>$.05). There was no significant difference in the food hygiene practices of food handlers based on level of education $(P=>.05)$. The study recommended among others, that managers of food business establishments should frequently supervise the hygienic condition under which food handlers working for them prepare food.
\end{abstract}

Keywords: Food Hygiene; Food handlers; Knowledge; Practice

\section{Introduction}

Assessing the knowledge and practice of food hygiene among food handlers have become important due to increase in reports of health problems associated with poor food hygiene. Globally, poor food hygiene knowledge and practices have been observed among some commercial and domestic food handlers, resulting to increased food-borne disease burden. According to World Health Organization (WHO) (2015), in an estimated 600 million world population, almost 1 in 10 people fall sick after eating contaminated food and 420,000 die every year. World Health Organization also reported that children under 5 years of age have 40 per cent of the food-borne disease burden, with 125,000 deaths every year. The implication for this could be that there are still food handlers who do not possess adequate knowledge of food hygiene and therefore cannot practice what they do not know. According to Bas, Ersun, and Kivanc (2006), food handlers often lack knowledge regarding basic food hygiene (critical temperatures of hot or cold ready-to-eat foods, acceptable refrigerator temperature ranges, and cross-contamination etc.).

Similarly, other studies have examined food hygiene knowledge and practices in Nigeria due to the alarming reports emanating from different parts of the world. In Nigeria, poor practice of food 
hygiene was reported in Nigeria's Federal Capital Territory (Ifeadike, Ironkwe, Adogu, \& Nnebue, 2014). Musa and Akande (2003) observed that up to 54 per cent of food handlers examined reuse water repeatedly for washing and cleaning without changing. Emmanuel, et al. (2015) reported that knowledge and practice of food hygiene and safety was low among food vendors and a significant proportion of them were not trained in food handling and preparation. Okojie, Wagbatsoma, and Ighoroge (2005) reported that there was a predominantly poor food handling in Nigerian. Jatau and Dangbin (2013) noted that food handlers in Plateau State possessed low knowledge on food hygiene and prevention of poisoning. Ofuebe (2015) discovered that some food vendors do not stay away from work during illness. They continue to work as long as the illness will allow them. Failure to observe sound food hygiene principles could have dire consequences. It could result in food poisoning and food-borne diseases such as cholera, diarrhoea and typhoid. Estimates reveal that as much as 70 percent of diarrhoeal diseases in developing countries are believed to be of foodborne origin (Emmanuel et al., 2015). It is therefore, important, to adhere to food hygiene principles.

Food hygiene is one of the three aspects of hygiene, with the others being environmental and personal hygiene. Food hygiene is a conscious effort to keep food safe from potential contamination and protect the health of consumers. defined food hygiene as all the practical measures involved in keeping food safe and wholesome through all the stages of production to point of sale or consumption (White, 2006; FAO \& WHO, 2009). White further explained that the implications of this definition are that food should be protected from spoilage and harmful microorganisms, harmful microorganisms present in food should be prevented from multiplying to an extent which would result in illness to consumers, harmful bacteria in the food should be destroyed by thorough cooking or processing, and that food should be safe, sound and wholesome when it reaches the consumer.

Failing to adhere to food hygiene principles throughout these stages may result in varying degrees of health problems. When this happens, food handlers can serve as channels for the transmission of harmful microbes. Food handlers play a major role in transmitting pathogens passively from contaminated sources such as transmitting pathogens from raw meat to a ready to eat food. Food handlers may carry some human specific food-borne pathogens such as Hepatitis A, typhoidal Salmonella, Staphylococcus aureus and Shigella species in their hands, cuts or sores, mouth, skin and hair (Adams \& Moss, 2008).

\section{Purpose of the Study}

The purpose of the study was to ascertain the food hygiene knowledge and practices among food handlers in University of Nigeria, Nsukka Campus. Specifically, the study determined:

1. level of knowledge of food hygiene possessed by food handlers based on level of education.

2. proportion of food handlers who practice food hygiene based on level of education.

\section{Research Questions}

Two research questions guided the study.

1. What is the level of knowledge of food hygiene possessed by food handlers based on level of education?

2. What is the proportion of food handlers that practice food hygiene based on level of education?

\section{Hypotheses}

Two hypotheses were postulated and tested at .05 level of significance.

$\mathrm{Ho}_{1}$. There is no significant difference in the level of knowledge of food hygiene possessed by food handlers based on level of education

$\mathrm{Ho}_{2}$. There is no significant difference in the proportion of food handlers that practice food hygiene based on level of education. 


\section{Methodology}

Area of the study: The study was conducted in University of Nigeria, Nsukka (UNN) Campus (the main campus). It is located in the heart of Nsukka town. In the campus, there are many vendors and restaurants, where various food handlers (mostly cooks and waiters) work. It was chosen as the location of study, because the researchers observed that the students and staff, who make up a greater part of the community's population, depend on food service establishments or food vendors for food.

Design of the study: The descriptive cross-sectional survey design was used in order to achieve the purpose of the study.

Population for the study: The population for the study consisted of all food handlers in University of Nigeria, Main Campus, including cooks and waiters. A total of 165 food handlers were identified and used for the study. Obtaining the number of all food handlers in the University of Nigeria from a registry body was not possible as not all of them are approved to sell or work in food service establishments that are registered with the Students Affairs Department of the Campus at the time of this study. The 165 food handlers were involved in the study, so, there was no sampling.

Instrument for data collection: The questionnaire was used for data collection. It was developed based on literature review and the specific objectives of the study. The instrument was face-validated by three health education experts. The reliability of the instrument was also established using split-half method. The Spearman's rank order correlation formula was used to compute the reliability of the instrument and a coefficient of .80 was yielded, which was adjudged high enough for use in the study.

Data collection techniques: A total of 165 copies of the questionnaire were administered by hand. Only 160 copies were however, duly completed and returned. This represents $97 \%$ return rate.

Data analysis techniques: Data were analyzed using frequencies and percentages. A proportion of $0-19 \%$ was interpreted as very low, $20-39 \%$ as low, $40-59 \%$ as moderate or average, $60-79$ as high and $80 \%$ and above as very high. The null hypotheses were tested using Chi-square $\left(X^{2}\right)$ statistic at .05 level of significance.

\section{Results}

Table 1: Percentage (\%) Responses on Knowledge of Food Hygiene Possessed by Food Handlers Based on Level of Education $(n=160)$

\begin{tabular}{|c|c|c|c|c|c|c|c|c|c|}
\hline \multirow[t]{2}{*}{$\mathbf{S} / \mathbf{N}$} & \multirow[t]{2}{*}{ Items } & \multirow{2}{*}{$\begin{array}{l}\text { No formal } \\
\text { education } \\
(n=2) \\
f(\%)\end{array}$} & \multicolumn{3}{|c|}{$\begin{array}{l}\text { Primary } \\
\text { education } \\
(n=9)\end{array}$} & \multicolumn{2}{|c|}{$\begin{array}{l}\text { Secondary } \\
\text { education } \\
(n=113)\end{array}$} & \multicolumn{2}{|c|}{$\begin{array}{l}\text { Tertiary } \\
\text { education } \\
(n=36)\end{array}$} \\
\hline & & & D & $f(0)$ & D & $f(\%)$ & D & $f(\%)$ & D \\
\hline 1 & $\begin{array}{l}\text { Food items exposed or not covered properly are not safe for human } \\
\text { consumption }\end{array}$ & $2(100.0)$ & $\mathrm{VH}$ & $7(77.8)$ & $\mathrm{H}$ & $111(98.2)$ & VH & $29(80.6)$ & $\mathrm{VH}$ \\
\hline 2 & $\begin{array}{l}\text { Food items packed in torn or leaking containers are not safe for human } \\
\text { consumption }\end{array}$ & $2(100.0)$ & $\mathrm{VH}$ & $9(100.0)$ & $\mathrm{VH}$ & $105(92.9)$ & VH & $29(80.6)$ & $\mathrm{VH}$ \\
\hline 3 & Food items past expiration date should not be bought & $2(100.0)$ & $\mathrm{VH}$ & $7(77.8)$ & $\mathrm{H}$ & $103(91.2)$ & $\mathrm{VH}$ & $34(94.4)$ & $\mathrm{VH}$ \\
\hline 4 & Food should be stored in refrigerators rather than in pots/other utensils & $2(100.0)$ & $\mathrm{VH}$ & $9(100.0)$ & $\mathrm{VH}$ & 105 (92.9) & VH & 32(88.9) & $\mathrm{VH}$ \\
\hline 5 & Raw food should be separated from cooked food during storage & $2(100.0)$ & $\mathrm{VH}$ & $7(77.8)$ & $\mathrm{H}$ & $103(91.2)$ & $\mathrm{VH}$ & $34(94.4)$ & $\mathrm{VH}$ \\
\hline 6 & Perishable food should be refrigerated within 2 hours & $2(100.0)$ & VH & $7(77.8)$ & $\mathrm{H}$ & $91(80.5)$ & $\mathrm{VH}$ & $32(88.9)$ & VH \\
\hline 7 & $\begin{array}{l}\text { Cooks should wash their hands properly with soap \& water before } \\
\text { cooking }\end{array}$ & $2(100.0)$ & VH & $7(77.8)$ & $\mathrm{H}$ & $113(100.0)$ & VH & $36(100.0)$ & $\mathrm{VH}$ \\
\hline 8 & $\begin{array}{l}\text { Cooks should wash their hands with soap and water after using the } \\
\text { toilet, sneezing, coughing or touching money while handling food to } \\
\text { avoid contamination. }\end{array}$ & $2(100.0)$ & VH & $9(100.0)$ & VH & $113(100.0)$ & VH & $36(100.0)$ & VH \\
\hline 9 & $\begin{array}{l}\text { Cooks should wash their hands with soap and water between handling } \\
\text { raw food and cooked food to avoid cross contamination. }\end{array}$ & $2(100.0)$ & VH & $7(77.8)$ & $\mathrm{H}$ & 111 (98.2) & $\mathrm{VH}$ & $36(100.0)$ & $\mathrm{VH}$ \\
\hline 10 & Surfaces and utensils should be cleaned before and after use & $2(100.0)$ & $\mathrm{VH}$ & $9(100.0)$ & $\mathrm{VH}$ & $105(92.9)$ & $\mathrm{VH}$ & $36(100.0)$ & $\mathrm{VH}$ \\
\hline 11 & $\begin{array}{l}\text { Covering of refuse bins (dust bins) and cleaning the cooking } \\
\text { surroundings is useful for food hygiene }\end{array}$ & $2(100.0)$ & $\mathrm{VH}$ & $9(100.0)$ & $\mathrm{VH}$ & $105(92.2)$ & VH & $36(100.0)$ & $\mathrm{VH}$ \\
\hline 12 & $\begin{array}{l}\text { Pipe borne water and water from underground wells are safer than } \\
\text { water from tankers and streams for cooking }\end{array}$ & $2(100.0)$ & VH & $7(77.8)$ & $\mathrm{H}$ & $94(83.2)$ & $\mathrm{VH}$ & $27(75.0)$ & $\mathrm{H}$ \\
\hline 13 & Cooks should reheat leftover food thoroughly before they are served. & $2(100.0)$ & VH & $9(10$ & $\mathrm{VH}$ & $101(8$ & $\mathrm{VH}$ & $32(88$ & $\mathrm{VH}$ \\
\hline 14 & Unwrapped cooked food should not be handled with bare hands & $2(100.0)$ & $\mathrm{VH}$ & $9(100.0)$ & VH & $97(85.8)$ & VH & $36(100.0)$ & $\mathrm{VH}$ \\
\hline 15 & $\begin{array}{l}\text { Waiters/waitresses should wear hair net/cap, gloves and apron while } \\
\text { serving food }\end{array}$ & $2(100.0)$ & VH & $9(100.0)$ & $\mathrm{VH}$ & 103 (91.2) & $\mathrm{VH}$ & $34(94.4)$ & $\mathrm{VH}$ \\
\hline 16 & $\begin{array}{l}\text { Waiters/waitresses should not come for work when they have signs } \\
\text { and symptoms of sickness to avoid exposing customers to health } \\
\text { problems }\end{array}$ & $2(100.0)$ & VH & $9(100.0)$ & VH & 107 (94.7) & $\mathrm{VH}$ & $30(83.3)$ & VH \\
\hline 17 & $\begin{array}{l}\text { Waiters/waitresses should not sneeze or cough directly over food } \\
\text { Overall (\%) }\end{array}$ & $\begin{array}{l}2(100.0) \\
100.0\end{array}$ & $\begin{array}{l}\text { VH } \\
\text { VH }\end{array}$ & $\begin{array}{l}9(100.0) \\
90.8\end{array}$ & $\begin{array}{l}\text { VH } \\
\text { VH }\end{array}$ & $\begin{array}{c}107(94.7) \\
92.3\end{array}$ & $\begin{array}{l}\text { VH } \\
\text { VH }\end{array}$ & $\begin{array}{c}36(100.0) \\
92.3\end{array}$ & $\begin{array}{l}\text { VH } \\
\text { VH }\end{array}$ \\
\hline
\end{tabular}


Data in Table 1 show that regardless of level of education, food handlers had very high level of knowledge of food hygiene knowledge. The data imply that all the respondents $(100 \%)$ who have no formal education were knowledgeable about food hygiene, 90.8 per cent of those who have primary education, 92.3 per cent of those who have secondary education and 92.3 per cent of those who have tertiary education had good knowledge of food hygiene.

Table 2: Proportion of Food Handlers that Practice Food Hygiene Based on Level of Education $(n=160)$

\begin{tabular}{|c|c|c|c|c|c|c|c|c|c|}
\hline \multirow{2}{*}{\multicolumn{2}{|c|}{ S/N Items }} & \multicolumn{2}{|c|}{$\begin{array}{c}\text { No formal } \\
\text { education }(n=2)\end{array}$} & \multicolumn{2}{|c|}{$\begin{array}{c}\text { Primary } \\
\text { education }(n=9)\end{array}$} & \multicolumn{2}{|c|}{$\begin{array}{c}\text { Secondary } \\
\text { education }(n=113)\end{array}$} & \multicolumn{2}{|c|}{$\begin{array}{c}\text { Tertiary } \\
\text { education }(n=36)\end{array}$} \\
\hline & & $f(\%)$ & D & $\mathbf{f}(\%)$ & D & $\mathbf{f}(\%)$ & D & $\mathbf{f}(\%)$ & D \\
\hline$\overline{1}$ & Buy food items exposed to contamination & $0(0.0)$ & $\mathrm{VL}$ & $4(44.4)$ & $\mathrm{M}$ & $49(43.4)$ & $\mathrm{M}$ & $19(52.8)$ & $\mathrm{M}$ \\
\hline 2 & Avoid buying food items packed in torn or leaking containers. & $2(100.0)$ & $\mathrm{VH}$ & $7(77.8)$ & $\mathrm{H}$ & $99(87.6)$ & $\mathrm{VH}$ & $26(72.2)$ & $\mathrm{H}$ \\
\hline 3 & Buy food items past expiration. & $0(0.0)$ & VL & $6(66.7)$ & $\mathrm{H}$ & $51(45.1)$ & M & $25(69.4)$ & $\mathrm{H}$ \\
\hline 4 & Store food in pots/other utensils rather than in refrigerators & $0(0.0)$ & $\mathrm{VL}$ & $4(44.4)$ & M & $55(48.7)$ & M & $23(63.9)$ & $\mathrm{H}$ \\
\hline 5 & Separated raw food from cooked food during storage & $2(100.0)$ & $\mathrm{VH}$ & $5(55.6)$ & M & $85(75.2)$ & $\mathrm{H}$ & $31(86.1)$ & VH \\
\hline 6 & Refrigerated perishable food within 2 hours. & $2(100.0)$ & VH & $9(100.0)$ & VH & $85(75.2)$ & $\mathrm{H}$ & $21(58.3)$ & $\mathrm{M}$ \\
\hline 7 & Wash my hands properly with soap \& water before cooking. & $2(100.0)$ & VH & $9(100.0)$ & VH & $103(91.2)$ & VH & $34(94.4)$ & $\mathrm{VH}$ \\
\hline 8 & $\begin{array}{l}\text { Wash my hands with soap and water after using the toilet, } \\
\text { sneezing, coughing or touching money while handling food. }\end{array}$ & $2(100.0)$ & VH & $7(77.8)$ & $\mathrm{H}$ & $103(91.2)$ & VH & $32(88.9)$ & VH \\
\hline 9 & $\begin{array}{l}\text { Wash my hands with soap and water between handling raw food } \\
\text { and cooked food. }\end{array}$ & $2(100.0)$ & VH & $7(77.8)$ & $\mathrm{H}$ & $101(89.4)$ & VH & $32(88.9)$ & VH \\
\hline 10 & Clean surfaces and utensils before and after use. & $2(100.0)$ & $\mathrm{VH}$ & $7(77.8)$ & $\mathrm{H}$ & $108(95.6)$ & $\mathrm{VH}$ & $36(100.0)$ & $\mathrm{VH}$ \\
\hline 11 & Cover refuse bins (dust bins) and clean my cooking surroundings. & $2(100.0)$ & VH & $5(55.6)$ & M & 105 & VH & $31(86.1)$ & $\mathrm{VH}$ \\
\hline 12 & $\begin{array}{l}\text { Cook with pipe borne water and water from underground wells } \\
\text { rather than water from tankers and streams. }\end{array}$ & $2(100.0)$ & $\mathrm{VH}$ & $9(100.0)$ & VH & $96(85.0)$ & VH & $20(55.6)$ & M \\
\hline 13 & Reheat leftover food thoroughly before they are served & $2(100.0)$ & $\mathrm{VH}$ & $7(77.8)$ & $\mathrm{H}$ & $104(92.0)$ & $\mathrm{VH}$ & $32(88.9)$ & $\mathrm{VH}$ \\
\hline 14 & Handle unwrapped cooked food with bare hands. & $0(0.0)$ & VL & $4(44.4)$ & M & $51(45.1)$ & M & $25(69.4)$ & $\mathrm{H}$ \\
\hline 15 & Wear hair net/cap, gloves and apron while serving food & $2(100.0)$ & $\mathrm{VH}$ & $7(77.8)$ & $\mathrm{H}$ & $101(89.4)$ & $\mathrm{VH}$ & $17(47.2)$ & M \\
\hline 16 & I come for work when I have signs and symptoms of sickness & $0(0.0)$ & VL & $4(44.4)$ & M & $62(54.9)$ & M & $25(69.4)$ & $\mathrm{H}$ \\
\hline 17 & I sneeze or cough directly over food. & $0(0.0)$ & VL & $8(88.9)$ & $\mathrm{VH}$ & $68(60.2)$ & $\mathrm{H}$ & $34(94.4)$ & $\mathrm{VH}$ \\
\hline & Overa & 64.7 & $\mathrm{H}$ & 71.2 & H & 74.2 & H & 75.7 & H \\
\hline
\end{tabular}

Table 2 shows that a high proportion $(64.7 \%)$ of food handlers practice food hygiene principle irrespective of their level of education. However, a slightly higher proportion of food handlers that have tertiary education $(75.7 \%)$ practised food hygiene principles more than those that have secondary education $(74.2 \%)$, those that have primary education $(71.2 \%)$ and those that have no formal education (64.7). Regardless of level of education, a very high proportion of food handlers indicated washing their hands properly with soap and water before cooking.

Table 3: Chi-Square $\left(x^{2}\right)$ Analysis of No Significant Difference in the Level of Knowledge of Food Hygiene Possessed by Food Handlers Based on Level of Education $(n=160)$

\begin{tabular}{|c|c|c|c|c|c|c|c|c|c|c|c|c|}
\hline & \multirow{3}{*}{ Item statement } & \multicolumn{2}{|c|}{$\begin{array}{l}\text { No formal } \\
\text { education } \\
\quad(n=2)\end{array}$} & \multicolumn{2}{|c|}{$\begin{array}{l}\text { Primary } \\
\text { education } \\
(n=9)\end{array}$} & \multicolumn{2}{|c|}{$\begin{array}{l}\text { Secondary } \\
\text { education }(n=113)\end{array}$} & \multicolumn{2}{|c|}{$\begin{array}{c}\text { Tertiary } \\
\text { education }(n=36)\end{array}$} & \multirow[b]{3}{*}{$\mathbf{X}^{2-\text { cal }}$} & \multirow{3}{*}{\multicolumn{2}{|c|}{ df $\begin{array}{c}P \text { - } \\
\text { value }\end{array}$}} \\
\hline & & CR & IR & CR & IR & CR & IR & CR & IR & & & \\
\hline & & $O(E)$ & $O(E)$ & $O(E)$ & $O(E)$ & $O(E)$ & $\mathrm{O}(\mathrm{E})$ & $\mathrm{O}(\mathrm{E})$ & $\mathrm{O}(\mathrm{E})$ & & & \\
\hline 1 & $\begin{array}{l}\text { Food items exposed or not covered properly are not safe } \\
\text { for human consumption }\end{array}$ & $2(1.9)$ & $0(0.1)$ & $7(8.4)$ & $2(0.6)$ & 111(105.2) & $2(7.8)$ & $29(33.5)$ & $7(2.5)$ & 1 & 23 & .001 \\
\hline 2 & $\begin{array}{l}\text { Food items packed in torn or leaking containers are not } \\
\text { safe for human consumption }\end{array}$ & $2(1.8)$ & $0(0.2)$ & $9(8.2)$ & $0(0.8)$ & 105 (102.4) & 8 (10.6) & $29(32.6)$ & $7(3.4)$ & 6.135 & 3 & .105 \\
\hline 3 & Food items past expiration date should not be bought & $2(1.8)$ & $0(0.2)$ & $7(8.2)$ & $2(0.8)$ & $103(103.1)$ & $10(9.9)$ & $34(32.9)$ & $2(3.2)$ & 2.699 & 3 & .440 \\
\hline 4 & $\begin{array}{l}\text { Food should be stored in refrigerators rather than in } \\
\text { pots/other utensils }\end{array}$ & $2(1.9)$ & $0(0.2)$ & $9(8.3)$ & $0(0.7)$ & 105 (104.5) & $8(8.5)$ & $32(33.3)$ & $4(2.7)$ & 1.597 & 3 & .660 \\
\hline 5 & $\begin{array}{l}\text { Raw food should be separated from cooked food during } \\
\text { storage }\end{array}$ & $2(1.8)$ & $0(0.2)$ & $7(8.2)$ & $2(0.8))$ & $103(103.1)$ & $10(9.9))$ & $34(32.9)$ & $2(3.2)$ & 2.699 & 3 & .440 \\
\hline 6 & Perishable food should be refrigerated within 2 hours & $2(1.7)$ & $0(0.4)$ & $7(7.4)$ & $2(1.6)$ & $91(93.2)$ & $22(19.8)$ & $32(29.7)$ & $4(6.3)$ & 1.885 & 3 & .597 \\
\hline 7 & $\begin{array}{l}\text { Cooks should wash their hands properly with soap \& } \\
\text { water before cooking } \\
\text { Cooks should wash their hands with soap and water }\end{array}$ & $2(2.0)$ & $0(0.0)$ & $7(8.9)$ & $2(0.1)$ & $113(111.6)$ & $0(1.4)$ & $36(35.6)$ & $0(0.5)$ & 33.980 & 3 & .000 \\
\hline 8 & $\begin{array}{l}\text { after using the toilet, sneezing, coughing or touching } \\
\text { money while handling food to avoid contamination. } \\
\text { Cooks should wash their hands with soap and water }\end{array}$ & $2(2.0)$ & $0(0)$ & $9(9.0)$ & $0(0)$ & $113(113.0)$ & $0(0)$ & $36(36.0)$ & $0(0)$ & - & 3 & - \\
\hline 9 & $\begin{array}{l}\text { between handling raw food and cooked food to avoid } \\
\text { cross contamination. }\end{array}$ & $2(2.0)$ & $0(.01)$ & $7(8.8)$ & $2(0.2)$ & $111(110.2)$ & $2(2.8)$ & $36(35.1)$ & $0(0.9)$ & 15.583 & 33 & .001 \\
\hline & $\begin{array}{l}\text { Surfaces and utensils should be cleaned before and } \\
\text { after use }\end{array}$ & $2(1.9)$ & $0(0.1)$ & $9(8.6)$ & $0(0.5)$ & 105 (107.4) & $8(5.7)$ & $36(34.2)$ & $0(1.8)$ & 3.503 & 3 & .320 \\
\hline & $\begin{array}{l}\text { Covering of refuse bins (dust bins) and cleaning the } \\
\text { cooking surroundings is useful for food hygiene. }\end{array}$ & $2(1.9)$ & $0(0.1)$ & $9(8.6)$ & $0(0.5)$ & 105 (107.4) & $8(5.7)$ & $36(34.2)$ & $0(1.8)$ & 3.503 & 3 & .320 \\
\hline
\end{tabular}


12 Pipe borne water and water from underground wells are $\quad 2(1.6) \quad 0(0.4) 7(7.3) 2(1.7) \quad 94(91.8) \quad 19(21.2) 27(29.3) \quad 9(6.8) \quad 1.734 \quad 3 \quad .629$

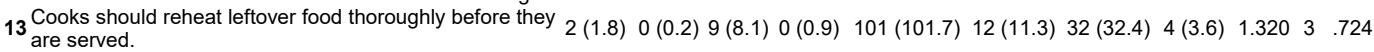

14 Unwrapped cooked food should not be handled with $\quad 2(1.8) 0(0.2) 9(8.1) 0(0.9) \quad 97(101.7) \quad 16(11.3) 36(32.4) 0(3.6) 7.3943 \quad .060$

Waiters/waitresses should wear hair net/cap, gloves and

15 apron while serving food

Waiters/waitresses should not come for work when they

16 have signs and symptoms of sickness to avoid exposing $2(1.9) \quad 0(0.2) 9(8.3) 0(0.7) \quad 107(104.5) \quad 6(2.7) \quad 30(33.3) 6(2.7) \quad 6.034 \quad 3 \quad .110$ customers to health problems

17 Waiters/waitresses should not sneeze or cough directly $\quad 2(1.9) \quad 0(0.1) 9(8.7) 0(0.3) \quad 107(108.8) \quad 6(4.2) \quad 36(34.7) \quad 0(1.4) \quad 2.593 \quad 3 \quad .459$ over food Overall $\mathrm{X}^{2}$

Key: $\mathrm{CR}$ = Correct Response; IR = Incorrect Response; O = Observed frequency; $\mathrm{E}$ = Expected frequency.

$\begin{array}{lll}6.812 & 3 & .348\end{array}$

Data in Table 3 show the overall chi-square value with the corresponding P-value for hypothesis of no significant difference in the level of knowledge of food hygiene possessed by food handlers based on level of education $\left(X^{2}\right.$ cal $\left.=6.812, p=.348>.05\right)$. Since the $P$-value was greater than .05 level of significance at 3 degree of freedom, the hypothesis of no significant difference was therefore not rejected. This implies that no significant difference existed in the level of knowledge of food hygiene possessed by food handlers based on level of education.

Table 4: Chi-Square Analysis of No Significant Difference in the Proportion of Food Handlers that Practice Food Hygiene Based on Level of Education $(n=160)$

\begin{tabular}{|c|c|c|c|c|c|c|c|c|c|c|c|}
\hline S/N Item & \multicolumn{2}{|c|}{$\begin{array}{l}\text { No formal } \\
\text { education } \\
\quad(n=2)\end{array}$} & \multicolumn{2}{|c|}{$\begin{array}{c}\text { Primary } \\
\text { education } \\
(n=9)\end{array}$} & \multicolumn{2}{|c|}{$\begin{array}{l}\text { Secondary education } \\
\qquad(n=113)\end{array}$} & \multicolumn{2}{|c|}{$\begin{array}{l}\text { Tertiary education } \\
\qquad(\mathrm{n}=36)\end{array}$} & \multirow[b]{3}{*}{$\mathrm{X}^{2-\mathrm{cal}}$} & \multirow[b]{3}{*}{ df } & \multirow[b]{3}{*}{$\begin{array}{c}P- \\
\text { value }\end{array}$} \\
\hline & $\mathrm{CP}$ & IP & $\mathrm{CP}$ & IP & CP & IP & CP & IP & & & \\
\hline & $O(E)$ & $\mathrm{O}(\mathrm{E})$ & $O(E)$ & $O(E)$ & $O(E)$ & $O(E)$ & $O(E)$ & $O(E)$ & & & \\
\hline 20 buy food items exposed to contamination & $0(0.9)$ & $2(1.1)$ & $4(4.1)$ & $5(5.0)$ & $49(50.9)$ & $64(62.2)$ & $19(16.2)$ & $17(19.8)$ & 2.640 & 3 & .451 \\
\hline $\begin{array}{l}21 \text { avoid buying food items packed in torn or leaking } \\
\text { containers. }\end{array}$ & $2(1.7)$ & $0(0.3)$ & $7(7.5)$ & $2(1.5)$ & $99(94.6)$ & $14(18.4)$ & $26(30.2)$ & $10(65.9)$ & 5.377 & 3 & .146 \\
\hline 22 buy food items past expiration. & $0(1.0)$ & $2(1.0)$ & $6(4.6)$ & $3(4.4)$ & $51(57.9)$ & $62(55.1)$ & $25(18.5)$ & $11(17.6)$ & 9.421 & 3 & .024 \\
\hline $\begin{array}{l}23 \text { store food in pots/other utensils rather than in } \\
\text { refrigerators }\end{array}$ & $0(1.0)$ & $2(1.0)$ & $4(4.6)$ & $5(4.4)$ & $55(57.9)$ & $58(55.1)$ & $23(18.5)$ & $13(17.6)$ & 4.872 & 3 & .181 \\
\hline $\begin{array}{l}24 \text { separated raw food from cooked food during } \\
\text { storage }\end{array}$ & $2(1.5)$ & $0(0.5)$ & $5(6.9)$ & $4(2.1)$ & $85(86.9)$ & $28(26.1)$ & $31(27.7)$ & $5(8.3)$ & 4.804 & 3 & .187 \\
\hline 25 refrigerated perishable food within 2 hours. & $2(1.5)$ & $0(0.5)$ & $9(6.6)$ & $0(2.4)$ & $85(82.6)$ & $28(30.4)$ & $21(26.3)$ & $15(9.7)$ & 8.303 & 3 & .040 \\
\hline $\begin{array}{l}26 \text { wash my hands properly with soap \& water } \\
\text { before cooking. } \\
\text { wash my hands with soap and water after using }\end{array}$ & 2 (1.9) & $0(0.2)$ & $9(8.3)$ & $0(0.7)$ & 103(104.5) & $10(8.55)$ & 34 (33.3) & $2(2.7)$ & 1.385 & 3 & .709 \\
\hline $\begin{array}{l}27 \text { the toilet, sneezing, coughing or touching money } \\
\text { while handling food. }\end{array}$ & $2(1.8)$ & $0(0.2)$ & $7(8.1)$ & $2(0.9)$ & $103(101.7)$ & $10(11.3)$ & $32(32.4)$ & $4(3.6)$ & 1.932 & 3 & .587 \\
\hline $\begin{array}{l}28 \text { wash my hands with soap and water between } \\
\text { handling raw food and cooked food. }\end{array}$ & $2(1.8)$ & $0(0.2)$ & $7(8.0)$ & $2(1.0)$ & $101(100.3)$ & $12(12.7)$ & $32(32.0)$ & $4(4.1)$ & 1.384 & 3 & .709 \\
\hline 29 clean surfaces and utensils before and after use. & $2(1.9)$ & $0(0.1)$ & $7(8.6)$ & $2(0.4)$ & $108(108.1)$ & $5(4.9)$ & $36(34.4)$ & $0(1.6)$ & 8.591 & 3 & .035 \\
\hline $\begin{array}{l}30 \text { cover refuse bins (dust bins) and clean my } \\
\text { cooking surroundings. } \\
\text { cook with pipe borne water and water from }\end{array}$ & $2(1.8)$ & $0(0.2)$ & $5(8.0)$ & $4(1.0)$ & 105 (101.0) & $8(12.0)$ & $31(32.2)$ & $5(3.8)$ & 12.977 & 3 & .005 \\
\hline $\begin{array}{l}31 \text { underground wells rather than water from tankers } \\
\text { and streams. }\end{array}$ & $2(1.6)$ & $0(0.4)$ & $9(7.1)$ & $0(1.9)$ & $96(89.7)$ & $17(23.3)$ & $20(28.6)$ & $16(7.4)$ & 17.484 & 3 & .001 \\
\hline $\begin{array}{l}32 \text { reheat leftover food thoroughly before they are } \\
\text { served }\end{array}$ & $2(1.8)$ & $0(0.2)$ & $7(8.2)$ & $2(0.8)$ & $104(102.4)$ & $9(10.6)$ & $32(32.6)$ & $4(3.4)$ & 2.348 & 3 & .503 \\
\hline 33 handle unwrapped cooked food with bare hands. & $0(1.0)$ & $2(1.0)$ & $4(4.5)$ & $5(4.5)$ & $51(56.5)$ & $62(56.5)$ & $25(18.0)$ & $11(18.0)$ & 8.626 & 3 & .035 \\
\hline $\begin{array}{l}34 \text { wear hair net/cap, gloves and apron while serving } \\
\text { food }\end{array}$ & $2(1.6)$ & $0(0.4)$ & $7(7.1)$ & $2(1.9)$ & 101 (89.7) & $12(23.3)$ & $17(28.6)$ & $19(7.4)$ & 30.177 & 3 & .000 \\
\hline $\begin{array}{l}35 \text { I come for work when I have signs and symptoms } \\
\text { of sickness }\end{array}$ & $0(1.1)$ & $2(0.9)$ & $4(5.1)$ & $5(3.9)$ & $62(64.3)$ & $51(49.7)$ & $25(20.5)$ & $11(15.5)$ & 5.709 & 3 & .127 \\
\hline $\begin{array}{l}36 \text { I sneeze or cough directly over food. } \\
\text { Overall } \mathbf{X}^{2}\end{array}$ & $0(1.4)$ & $2(0.6)$ & $8(6.2)$ & $1(2.8)$ & $68(77.7)$ & $45(35.3)$ & $34(24.8)$ & $2(11.3)$ & $\begin{array}{c}21.027 \\
8.650\end{array}$ & 3 & $\begin{array}{l}.000 \\
0.220\end{array}$ \\
\hline
\end{tabular}

Table 4 shows the overall chi-square value with the corresponding P-value for hypothesis of no significant difference in the food hygiene practices of food handlers based on level of education $\left(X^{2}\right.$ cal $=8.650, p=.220>.05)$. Since the P-value was greater than .05 level of significance at 3 degree of freedom, the hypothesis of no significant difference was therefore not rejected. This implies that no significant difference existed in the food hygiene practices of food handlers based on level of education. 


\section{Discussion}

Table 1 showed the overall percentage of food handlers' level of knowledge of food hygiene based on level of education. Irrespective of level of education, food handlers had very high knowledge of food hygiene, with those who have no formal education (100\%), those who have primary education $(90.8 \%)$, those who have secondary education $(92.3 \%)$ and those who have tertiary education $(92.3 \%)$. The result was expected. The influence of being in an environment of higher learning such as obtainable in the location of study must be responsible for this result. These findings are in consonance with the findings of Oghenekohwo (2015) who asserted that there was a high level of knowledge, awareness and application of food hygiene practices among food vendors in the Niger Delta University Communities. Similarly, Annor and Baiden (2011) asserted that food hygiene knowledge and attitudes of food handlers were satisfactory, however its practice was challenging. These findings are however, contradictory to Tang and Fong (2004) who reported that of 580 food handlers they studied, 79.0 per cent showed their need of more food hygiene education. Zain and Naing (2002) reported that $(48.4 \%)$ almost half of the food handlers studied had poor knowledge on food hygiene. The difference in findings could be attributed to environmental influence. This implies that health educators should direct more effort towards food handlers in non-educational environments. Tables 3 showed that there was no significant difference in the level of food hygiene knowledge possessed by food handlers based on level of education $\left(X^{2}=6.812, P=.348>.05\right.$ at $\left.3 \mathrm{df}\right)$. This was surprising as logically, with more education food handlers should be more informed about diverse matters including food hygiene. This is contrary to the findings of Sharif, Obaidat, \& AlDalalah (2013) who found that university education respondents had a higher score than primary education respondents on food handling. Again the difference could due to environmental influence. The implication of this finding is that level of education doesn't necessarily play a role in the level of food hygiene knowledge.

Table 2 showed that a slightly higher proportion of food handlers that have tertiary education $(75.7 \%)$ practiced sound food hygiene principles more than those that have secondary education $(74.2 \%)$, those that have primary education $(71.2 \%)$ and those that have no formal education $(64.7 \%)$. These findings were expected. This could be owing to the fact that knowledge has created confidence in them to actually adhere to practices. The findings were in conformity with the findings of Ofuebe (2015), who reported that most food vendors adhere to the principles of food hygiene recommended by World Health Organization. Similarly, Isara and Isah as cited by Igbokwe and Dibia (2015) reported that there was good practice of food hygiene and safety among respondents. The findings were however in contradiction to Samapundo, Climat, Xhaferi and Devlieghere (2014) who reported that majority of food handlers served food with bare hands and did not wash their hands after handling money. Similarly, Mulugeta and Bayeh (2012) reported that there were poor conditions and poor food hygiene practices of handlers. Muinde and Kuria (2005) also reported that about 85 per cent of food handlers prepared their foods in unhygienic conditions. Table 4 showed that there was no significant difference in the food hygiene practices of food handlers based on level of education $\left(X^{2}=8.650, P=.220>.05\right.$ at $3 \mathrm{df}$ ). This is not in line with the findings of Oghenekohwo (2015) who attributed the high level of food hygiene, knowledge, awareness and appliance of food vendors to their educational attainment. The implication is that the task of health educating food handlers should not be neglected for any reason.

\section{Conclusion}

Food hygiene knowledge was very high for food handlers of all levels of education. Food hygiene practices were high for food handlers of all levels of education. There was no significant difference in the level of food hygiene knowledge possessed by food handlers based on levels of education. There was no significant difference in the food hygiene practices of food handlers based on level of education. 


\section{Recommendations}

From the findings of this study, the following recommendations were therefore made.

1. Managers of food business establishments should frequently supervise the hygienic condition under which food handlers working for them prepare food. As well as constantly reward adherence to food hygiene principles.

2. The local and national authorities should set up a committee, which will frequently inspect the food hygiene practices of food handlers and vendors across the country.

3. A bill should be passed to the effect that only food handlers who have received adequate food hygiene training and have been certified should be allowed to work as food vendors or food handlers.

\section{References}

Adams, M. R., \& Moss, M. O. (2008). Food Microbiology, ( $3^{\text {rd }}$ ed.). Cambridge: The Royal Society of Chemistry.

Annor, G. A., \& Baiden, E. A. (2011). Evaluation of food hygiene knowledge attitudes and practices of food handlers in food businesses in Accra, Ghana. Food and Nutrition Sciences, 2, 830-836. doi:10.4236/fns.2011.28114

Bas, M. Ersun, A. S. \& Kivanc, G. (2006). The evaluation of food hygiene knowledge, attitudes, and practices of food handlers in food businesses in Turkey. Food Control, 17, 317-322. doi:10.1016/j.foodcont.2004.11.006

Emmanuel, A., Mangai, J. M., Kayong, E. A., Afoi, B. B., Goshit, J. D., Naman, K., \& Innocent, O. (2015). Assessment of practice of food safety and hygiene among food vendors within Jos North Local Government Area of Plateau State, Nigeria. International Journal of Medical and Health Research, 1 (2), 83-86.

Food and Agricultural Organization \& World Health Organization (2009). Codex Alimentarius: food hygiene basic text $\left(4^{\text {th }}\right.$ ed.). Retrieved from http://www.fao.org/docrep/012/a1552e00.pdf

Ifeadike, C. O., Ironkwe, O. C., Adogu, P. O., \& Nnebue, C. C. (2014). Assessment of the food hygiene practices of food handlers in the Federal Capital Territory of Nigeria. Tropical Journal of Medical Research, 17 (1), 10-15.

Igbokwe, C. C., Igbokwe, C. B. \& Dibia, S. I. C. (2015). Knowledge and practice of salmonellosis prevention among food handlers in restaurants in University of Nigeria Nsukka. International Journal of Human Kinetics, Health and Education, 1 (1), 26-34

Jatau, A., \& Dangbin, P. (2013). Knowledge and practices of food hygiene among food handlers in restaurants in Mazat district, Barking Ladi LGA, Plateau State, Nigeria. Nigerian Journal of Health Education, 17 (1), 238-250.

Muinde, O. K., \& Kuria, E. (2005). Hygienic and Sanitary practices of vendors of street foods in Nairobi, Kenya. African Journal of Food Agriculture and Nutritional Development, 5 (1), 1-13

Mulugeta, K., \& Bayeh, A. (2012). The sanitary conditions of food service establishments and food safety knowledge and practices of food handlers in Bahir Dar Town. Ethiopian Journal of Health Science, 22 (1), 27-35.

Musa, O. I., \& Akande, T. M. (2003). Food hygiene practices of food vendors in secondary schools in llorin. The Nigerian Postgraduate Medical Journal, 10 (3), 192-196.

Ofuebe, J. I. (2015). Food hygiene practices among food vendors in University of Nigeria, Nsukka Campus: implication for consumer protection. International Journal of Human Kinetics, Health and Education, 1 (1), 92-98.

Oghenekohwo, J. E. (2015). Pattern of food hygiene and environmental health practices among food vendors in Niger Delta University. European Journal of Food Science and Technology, 3 (1), 24-40.

Okojie, O. H., Wagbatsoma, V., A., \& Igboroge, A. D. (2005). An assessment of food hygiene among food handlers in a Nigerian University campus. Nigerian Postgraduate Medical Journal, 12 (2), 93-96.

Samapundo S., Climat R., Xhaferi R., \& Devlieghere F. (2014). Food safety knowledge, attitudes and practices of street food vendors and consumers in Port-au-Prince, Haiti. doi: 10.1016/j.foodcont.2014.09.010.

Sharif, L., Obaidat, M. M., \& Al-Dalalah, M. R. (2013). Food hygiene knowledge, attitudes and practices of the food handlers in the military hospitals. Food and Nutrition Sciences, 4, 245-251. Retrieved from http://dx.doi.org/10.4236/fns.2013.43033.

Tang, C. H., \& Fong, U. W. (2004). A survey of food hygiene knowledge and attitudes among Chinese food handlers in Fong Song Tong district. Asia-Pacific Journal of Public Health / Asia-Pacific Academic Consortium for Public Health, 16 (2), 121-125. 
White, R. (2006). Food hygiene - why bother? Retrieved from http://www.safefood.eu/SafeFood/media/SafefoodLibrary/Education/safefood\%20for\%20life/NI/section1.pdf World Health Organization (2015). Food safety. Retrieved from www.who.int/mediacentre/factsheets/fs399/en/

Zain \& Naing (2002). Socio-demographic characteristics of food handlers and their knowledge, attitude and practice towards food sanitation: a preliminary report. Southeast Asian Journal of Tropical Medical Public Health, 33 (2), 410-417. 